\title{
Atomic transitions and the first ionization potential of promethium determined by laser spectroscopy
}

\author{
Dominik Studer, ${ }^{1, *}$ Stephan Heinitz, ${ }^{2}$ Reinhard Heinke, ${ }^{1}$ Pascal Naubereit, ${ }^{1}$ Rugard Dressler ${ }^{3}$ Carlos Guerrero, ${ }^{4}$ Ulli Köster, ${ }^{5}$ \\ Dorothea Schumann, ${ }^{3}$ and Klaus Wendt ${ }^{1}$ \\ ${ }^{1}$ Institut für Physik, Johannes Gutenberg-Universität Mainz, 55128 Mainz, Germany \\ ${ }^{2}$ Belgian Nuclear Research Centre, SCK-CEN, 2400 Mol, Belgium \\ ${ }^{3}$ Paul-Scherrer Institut, 5232 Villigen, Switzerland \\ ${ }^{4}$ Departamento de Física Atómica, Molecular y Nuclear, Universidad de Sevilla, 41012 Sevilla, Spain \\ ${ }^{5}$ Institut Laue-Langevin, 38042 Grenoble, France
}

(Received 19 March 2019; published 26 June 2019)

\begin{abstract}
The atomic spectrum of neutral promethium has been studied extensively by laser resonance ionization spectroscopy. We report on more than 1000 atomic transitions in the blue and near infrared spectral ranges, most of them between high excited energy levels. As Rydberg convergences could not be assigned unambiguously in the dense spectrum at high excitation energies, the first ionization potential (IP) was determined via field ionization of weakly bound states within a static electric field. By applying the saddle-point model, a value of $\mathrm{IP}_{(\mathrm{Pm})}=45020.8(3) \mathrm{cm}^{-1}[5.58188(4) \mathrm{eV}]$ was derived, which confirms previous expectations of $45027(80)$ and $44985(140) \mathrm{cm}^{-1}$, which were obtained indirectly from lanthanide IP systematics.
\end{abstract}

DOI: 10.1103/PhysRevA.99.062513

\section{INTRODUCTION}

The year 2019 has been declared the International Year of the Periodic Table of Chemical Elements for the 150th anniversary of Mendeleev's discovery, which today comprises 118 elements. While most stable species are studied thoroughly, for a number of elements, which either have no stable isotopes or are produced only artificially, still today fundamental atomic properties have not been determined with satisfying precision or are entirely missing. These deficits also include the ionization potential (IP), i.e., the energy required to remove one electron from the neutral atom. In this sense we aim to shed light on the promethium case ( $\mathrm{Pm}, Z=61)$, which is the only exclusively radioactive lanthanide element. The longest-lived isotope of this element is ${ }^{145} \mathrm{Pm}$ with a $17.7-\mathrm{yr}$ half-life; however, the more commonly used isotope in the few practical applications of this element is ${ }^{147} \mathrm{Pm}$ with a half-life of $2.6 \mathrm{yr} .{ }^{147} \mathrm{Pm}$ is used in nuclear batteries [1], e.g., for space missions and as a $\beta$ source for thickness gauges [2]. ${ }^{142} \mathrm{Pm}$ $\left(T_{1 / 2}=40.5 \mathrm{~s}\right)$ in a so-called ${ }^{142} \mathrm{Sm} /{ }^{142} \mathrm{Pm}$ in vivo generator had been used for preclinical positron emission tomography at a Geneva hospital [3]. ${ }^{149} \mathrm{Pm}\left(T_{1 / 2}=2.2\right.$ days $)$ is a promising radiolanthanide for receptor-targeted radiotherapy [4] due to its emission of medium-energy $\beta$ rays and only a few disturbing gamma rays. It can be produced via neutron activation in non-carrier-added form $[5,6]$.

\footnotetext{
*dstuder@uni-mainz.de

Published by the American Physical Society under the terms of the Creative Commons Attribution 4.0 International license. Further distribution of this work must maintain attribution to the author(s) and the published article's title, journal citation, and DOI.
}

First spectroscopy studies of promethium were performed with milligram samples of ${ }^{147} \mathrm{Pm}$, corresponding to an activity in the terabecquerel range. These measurements revealed numerous resonance lines in the spectra of neutral (Pm I) and singly charged (Pm II) promethium [7,8], as well as hyperfine splittings of ${ }^{147} \mathrm{Pm}$ [9]. The most comprehensive works on Pm I energy levels and transitions to date are [7,10], which also account for the major part in spectroscopic data compilations, such as $[11,12]$. However, one should note that only well-assigned atomic transitions from the low-lying ${ }^{6} \mathrm{H}^{o}$ and ${ }^{6} \mathrm{~F}^{o}$ fine-structure multiplets with excitation energies of $E<10000 \mathrm{~cm}^{-11}$ are given in literature, leaving almost the upper half of the spectrum unexplored. Moreover, the first ionization potential of $\mathrm{Pm}$ has never been determined experimentally. Worden et al. [13] and Wendt et al. [14] report values of $45027(80) \mathrm{cm}^{-1}$ and $44985(140) \mathrm{cm}^{-1}$, respectively. Both of these results were obtained from a systematic interpolation of the IPs of all lanthanide elements. A direct measurement could confirm the underlying assumption of a linear trend in the IPs of lanthanide atoms above and below the half-filling of the $4 f$ shell. Considering that the IP has recently been measured up to $Z=103[15,16]$, it is even more remarkable that this fundamental property of Pm remains unknown. Figure 1 gives an overview of the present situation regarding the experimental uncertainties in the first ionization potential across the Periodic Table. With the exception of the transactinide elements, Pm marks the last gap where no experimental result is listed. Apart from the high specific radioactivity of $\mathrm{Pm}$ isotopes, this is a consequence of the

\footnotetext{
${ }^{1}$ As commonly used in spectroscopy, we give energies (and IP values) in units of $\mathrm{cm}^{-1}$, which can be converted to actual energy values by multiplication with $h c$. Here $1 \mathrm{~cm}^{-1}$ corresponds to $1.24 \times$ $10^{-4} \mathrm{eV}$.
} 


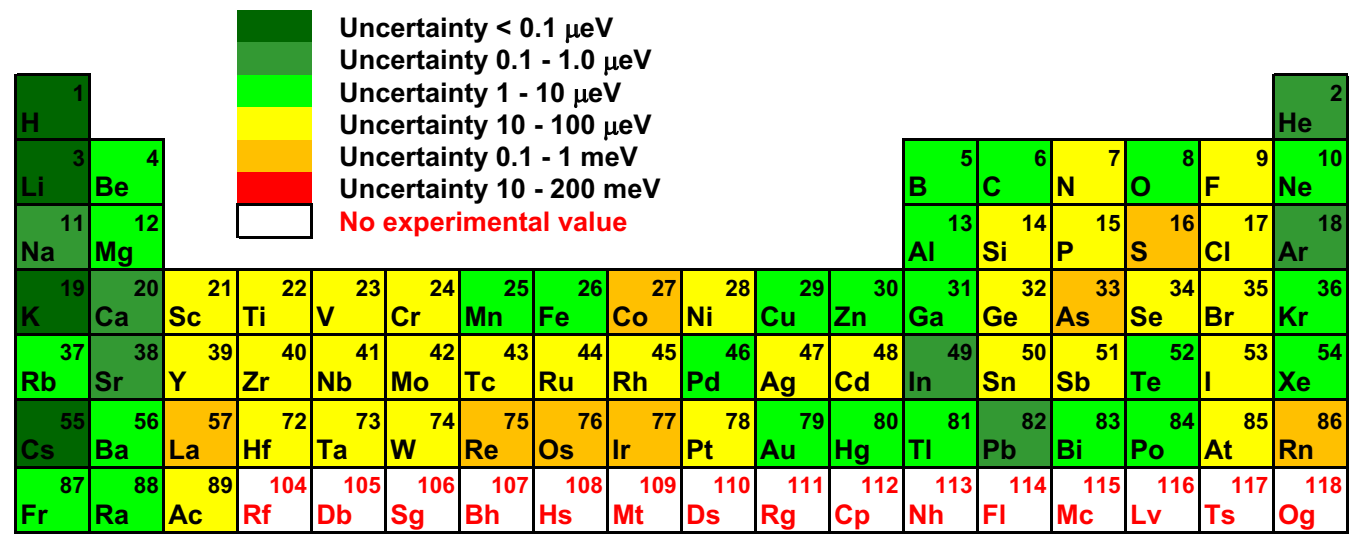

\begin{tabular}{|l|l|l|l|l|l|l|l|l|l|l|l|l|l|}
\hline $\mathrm{Ce}^{58}$ & $\mathrm{Pr}^{59}$ & $\mathrm{Nd}^{60}$ & $\mathrm{Pm}^{61}$ & $\mathrm{Sm}^{62}$ & $\mathrm{Eu}^{63}$ & $\mathrm{Gd}^{64}$ & $\mathrm{~Tb}^{65}$ & $\mathrm{Dy}^{66}$ & $\mathrm{Ho}^{67}$ & $\mathrm{Er}^{68}$ & $\mathrm{Tm}^{69}$ & $\mathrm{Yb}^{70}$ & $\mathrm{Lu}^{71}$ \\
\hline $\mathrm{Th}^{90}$ & $\mathrm{~Pa}^{91}$ & $\mathrm{U}^{92}$ & $\mathrm{~Np}^{93}$ & $\mathrm{Pu}^{94}$ & $\mathrm{Am}^{95}$ & $\mathrm{Cm}^{96}$ & $\mathrm{Bk}^{97}$ & $\mathrm{Cf}^{98}$ & $\mathrm{Es}^{99}$ & $\mathrm{Fm}^{100}$ & $\mathrm{Md}^{101}$ & $\mathrm{No}^{102}$ & $\mathrm{Lr}^{103}$ \\
\hline
\end{tabular}

FIG. 1. Periodic Table of Chemical Elements with a color coded display of the experimental uncertainty in the value of the first ionization potential for each element. In the case of Pm the upper half of the tile corresponds to the achieved precision in this work. A complete list of all values, uncertainties, and corresponding references is given in the Supplemental Material [17].

complex atomic spectra of midshell elements, most notably in the lanthanide and actinide series with an additional open $f$ subshell. Usually the analysis of Rydberg series allows a determination of the ionization potential with a precision in the range of $10^{-5} \mathrm{eV}$, as demonstrated for a number of radioactive elements, e.g., Tc [18], Ac [19], Po [20,21], At [22], or No [23]. However, the identification of Rydberg states becomes increasingly difficult with the occurrence of strong configuration mixing in complex atomic systems, up to a point where an unambiguous level assignment is not possible anymore. The spectrum may even exhibit chaotic behavior, as recently observed in $\mathrm{Pa}$ [24,25]. In this work we apply a complementary approach for the IP determination, based on dc electric field ionization of highly excited levels, which even benefits from high level density. The method was utilized by Köhler et al. [26] and Erdmann et al. [27] for the measurement of IPs of several actinide elements. We refined this approach for applicability at even lower counting statistics without a loss in precision.

\section{EXPERIMENTAL SETUP}

Our experiment is based on resonance ionization spectroscopy (RIS), which offers an excellent sensitivity for the investigation of minuscule sample amounts. The method relies on a stepwise photoionization process by pulsed laser radiation, providing an inherent elemental selectivity together with usually high efficiency.

For our measurements we used a sample of approximately $6 \times 10^{13}$ atoms of ${ }^{147} \mathrm{Pm}$ (corresponding to $15 \mathrm{ng}$ or $500 \mathrm{kBq}$ ). It was produced in the high-flux reactor at ILL Grenoble by neutron activation of highly ${ }^{146} \mathrm{Nd}$-enriched neodymium. The produced ${ }^{147} \mathrm{Nd}$ decays with a half-life of 11 days to ${ }^{147} \mathrm{Pm}$. The irradiated sample was dissolved in $7 M \mathrm{HNO}_{3}$ and underwent chemical purification via ion exchange chromatography at PSI Villigen in order to remove the macroscopic Nd component, as described in Ref. [28]. Part of this batch was repurified from the ${ }^{147} \mathrm{Sm}$ decay product using extraction chromatography. The purified ${ }^{147} \mathrm{Pm}$ solution was shipped to Mainz University for the spectroscopy measurements. The experimental apparatus is shown in Fig. 2. The principle of RIS is implemented with a hot cavity laser ion source coupled to a compact quadrupole mass spectrometer. The Pm solution is heated and crystallized on a $3 \times 3 \mathrm{~mm}^{2}$ titanium carrier foil (also serving as a reduction agent), which is then folded and introduced into a tubular graphite furnace with $37 \mathrm{~mm}$ length and $2.2 \mathrm{~mm}$ inner diameter. The furnace can be heated resistively with a current of up to $100 \mathrm{~A}$. At a temperature of approximately $850{ }^{\circ} \mathrm{C}$ the $\mathrm{Pm}$ is atomized [according to chemical equilibrium simulation (Outotec HSC Chemistry)] and irradiated by pulsed laser light. Laser-ionized species are extracted and accelerated by a set of three extraction electrodes $\left(U_{1}, U_{2}\right.$, and $\left.U_{3}\right)$. The ion beam is guided through a $90^{\circ}$ electrostatic quadrupole deflector to remove

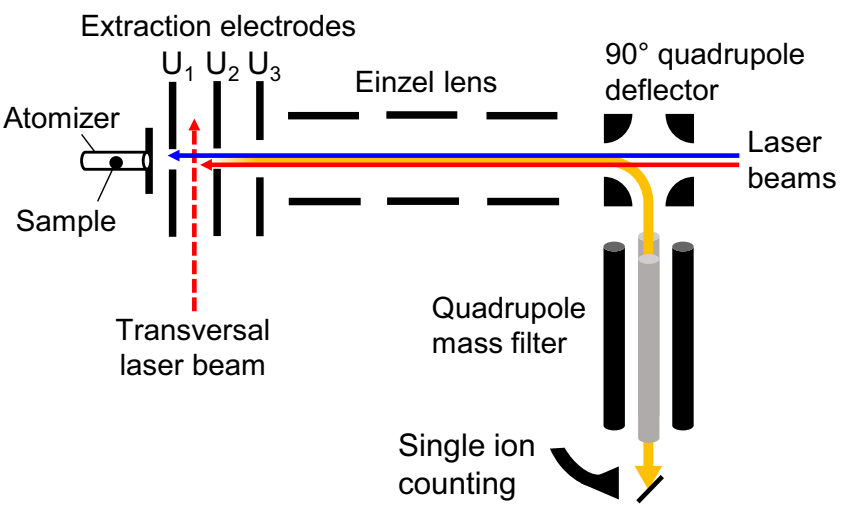

FIG. 2. Sketch of the atomic beam mass spectrometer with ion flight path (yellow) and laser beams in anticollinear (solid red and solid blue) and perpendicular crossed-beam (dashed red) geometry. The latter offers a spatially-well-defined laser-atom interaction region inside a homogeneous electric field between $U_{1}$ and $U_{2}$. 


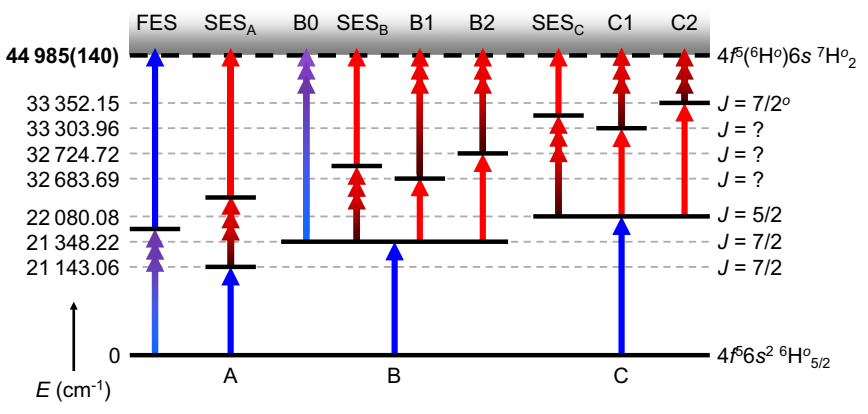

FIG. 3. Overview of investigated excitation schemes in Pm I. The respective scanning excitation step is indicated by a series of arrows. The notation for every scheme is given on top. The letters A, B, and $\mathrm{C}$ below define the schemes in a more general sense, referring to the respective first excitation step. The level energies for the first excited states are taken from the NIST database [11].

unspecific neutral background components and directed into an rf quadrupole mass filter. Singly charged ions of the desired mass are finally detected with a channel electron multiplier in single-ion-counting mode.

In standard operation all lasers are guided along the ion beam axis, ionizing the sample directly within the atomizer cavity. The alternative, transversal ionization geometry is discussed in Sec. IV. The laser system consists of three pulsed Ti:sapphire lasers. Each laser is pumped with 13-18 W of a $10-\mathrm{kHz}$ repetition rate $\mathrm{Nd}$ :YAG laser at $532 \mathrm{~nm}$ (Photonics Industries DM100-532). Under optimal conditions the average fundamental output of each Ti:sapphire laser reaches up to $5 \mathrm{~W}$, with a pulse length of 40-60 ns and a spectral linewidth of 5-8 GHz so that all Doppler classes within the hot atomic vapor are addressed. This type of laser system is also in operation at the majority of on-line radioactive ion beam facilities worldwide (for details see [29,30]). In our setup we extend the fundamental tuning range of 680-960 nm by second harmonic generation in an external beta barium borate (BBO) single-pass assembly with a conversion efficiency of approximately $10 \%$. Wide-range scans are performed with a dedicated spectroscopy Ti:sapphire laser. It is based on a diffraction grating for frequency selection, allowing modehop-free scanning operation by angle tuning of the grating. This laser type has an output power of approximately $2 \mathrm{~W}$ and a spectral linewidth of 2-5 GHz. A similar laser design is described in [31]. Scanning of the second harmonic is performed by manual phase-matching adjustment via the BBO tilting angle, while spatial beam walk-off is compensated with a system of position sensitive detectors and motorized mirrors (TEM Aligna Beamlock 2D). The fundamental frequency of each laser is monitored with a wavelength meter during all measurements (High Finesse WS6-600).

\section{BROADBAND LASER SPECTROSCOPY}

Several optical excitation ladders were investigated in order to probe the spectrum of Pm I and for the development of resonance ionization schemes. An overview of all schemes is given in Fig. 3, where a series of arrows indicates the scanned excitation step. In the following these schemes are abbreviated with the notation given on top in Fig. 3. Initially, a search for first excited states (scheme FES) was performed by scanning one laser from 408 to $473 \mathrm{~nm}$, along with a second laser at a fixed wavelength for nonresonant ionization from excited states. The spectrum contains 196 lines. In an independent scan with a sample of natural $\mathrm{Sm}$ we could identify 43 of those lines as parasitic resonances from the isobaric ${ }^{147} \mathrm{Sm}$ daughter nuclide of ${ }^{147} \mathrm{Pm}$, which cannot be separated in our mass filter. Most of the recorded Pm lines can be found in [10]. Our data show a systematic deviation of $\Delta \tilde{v}_{\mathrm{Pm}}=(-0.12)_{\mathrm{sys}}(4)_{\mathrm{stat}} \mathrm{cm}^{-1}$ from Pm literature values (the statistical error is inferred from the standard deviation). In the case of the observed Sm lines this deviation is $\Delta \widetilde{v}_{\mathrm{Sm}}=$ $(-0.11)_{\text {sys }}(4)_{\text {stat }} \mathrm{cm}^{-1}$ when comparing our results to energies given in the NIST database [11]. This systematic shift is well understood and arises from an imperfect synchronization in the data acquisition process, depending on the laser scan speed. In independent scans of the ground-state transitions in schemes $\mathrm{B}$ and $\mathrm{C}$ we could perfectly reproduce the literature values. Therefore, a correction of the shifts mentioned above was applied to the recorded data. In the Supplemental Material [17] we list the 32 previously unknown transitions. We refrain from giving associated energy levels in this case, as we cannot clearly determine the lower level energy. This is due to a thermal population of low-energy states, thus observed lines are not necessarily ground-state transitions. In fact, when comparing our spectrum with literature data, we find that the whole ${ }^{6} \mathrm{H}^{o}$ multiplet of altogether six levels with $5 / 2 \leqslant J \leqslant 15 / 2$ is considerably populated in the hot atomizer. At an average operating temperature of $1200^{\circ} \mathrm{C}$, the relative ${ }^{6} \mathrm{H}_{5 / 2}^{o}$ ground state population is at approximately $55 \%$. However, one should note that this state maintains leading percentage in the ensemble even at high temperatures of $2000^{\circ} \mathrm{C}$, making it the initial state of choice for efficient photoionization. As first excited states we chose the three odd-parity levels at 21 143.06, 21348.22 , and $22080.08 \mathrm{~cm}^{-1}$ for further investigation, because there are strong ground-state transitions leading to these levels. Moreover, these transitions fulfill the condition $2 \tilde{v}<$ IP, preventing a one-color, twophoton ionization process which would induce background in further spectroscopy measurements.

Second excitation steps (schemes $\mathrm{SES}_{\mathrm{A}}, \mathrm{SES}_{\mathrm{B}}$, and $\mathrm{SES}_{\mathrm{C}}$ ) were measured in the same manner, i.e., with the first excitation step fixed on the respective resonance and with the addition of a third, nonresonant step. We recorded 157 lines and 126 associated even-parity energy levels. Possible values for the total angular momentum are constrained by the selection rule $\Delta J=0, \pm 1$ for dipole transitions and may be further restricted whenever a state is visible in more than one excitation scheme. In the case of the excited state at $33352.15 \mathrm{~cm}^{-1}$ the total angular momentum was determined as $J=7 / 2$ by a measurement of the hyperfine structure of the $22080.08 \mathrm{~cm}^{-1} \rightarrow 33352.15 \mathrm{~cm}^{-1}$ transition. Details on these measurements are beyond the scope of the present work.

Some first and second step transitions were also analyzed with respect to their saturation behavior. Figure 4 shows the laser power influence on the line shape and the ion signal in excitation scheme $\mathrm{C} 2$. In the region of strong saturation the ion signal is almost independent of the laser power and the line profile is significantly broadened. The saturation power $P_{\text {sat }}$ is defined as the laser power where half of the 


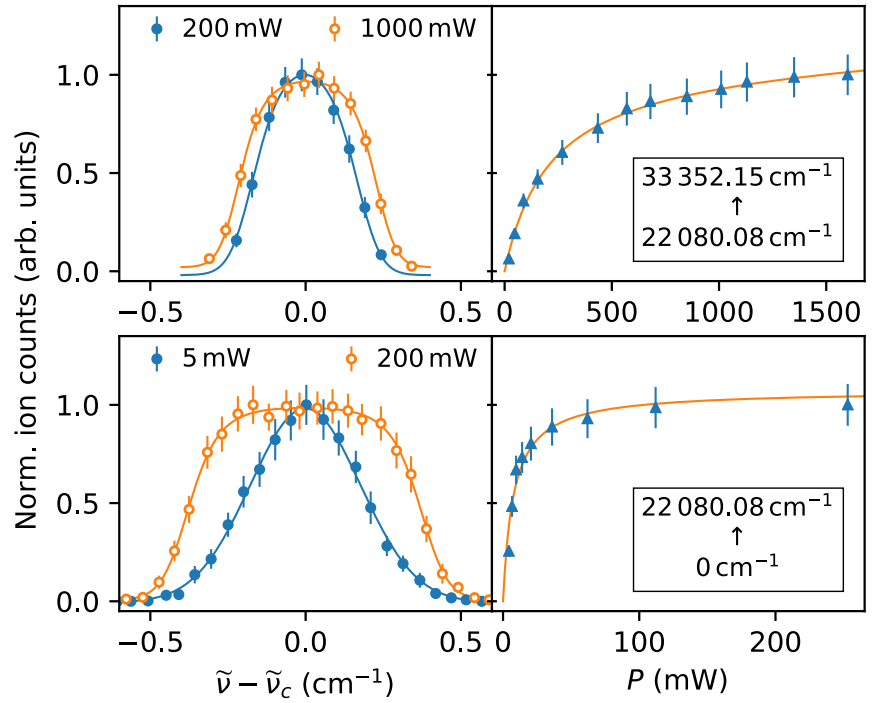

FIG. 4. Shown on the left are line profiles of the first step (bottom) and second step (top) transitions in excitation scheme C2 for different laser powers. Ion counts are normalized in order to emphasize the saturation broadening. Shown on the right is the laser power dependence of the ion signal.

maximum ion signal is reached. The saturation curve fit for the determination of $P_{\text {sat }}$ and the broadened line shape are discussed in detail in [32]. All saturation power measurements are summarized in Table I. For reasons of time, only a limited number of transitions could be investigated; however, it is clear that all these transitions can be easily saturated with the available laser power, which is favorable with regard to the ionization efficiency.

Finally, ionizing transitions were measured in the excitation schemes B0, B1, B2, C1, and C2. The high level density is similar in all spectra and only slightly varies with the signalto-background ratio in the different scans. A sample spectrum from excitation scheme C2 is shown in Fig. 5, containing 342 lines in a range of approximately $800 \mathrm{~cm}^{-1}$. An increase in the overall ion signal can be observed in the regions of the expected IP [13,14], which suggests that the linear trend in lanthanide IPs is indeed accurate. However, a more precise IP value may not be readily determined from these spectra. The nonresonant photoionization threshold is obscured due to the high level density and Rydberg states converging to the ${ }^{7} \mathrm{H}_{3}^{o}$
TABLE I. Saturation powers $P_{\text {sat }}$ for several investigated transitions with lower energy level $E_{l}$ and upper level $E_{u}$.

\begin{tabular}{lccc}
\hline \hline Scheme & $E_{l}\left(\mathrm{~cm}^{-1}\right)$ & $E_{u}\left(\mathrm{~cm}^{-1}\right)$ & $P_{\text {sat }}(\mathrm{mW})$ \\
\hline A & 0 & 21143.06 & $3(1)$ \\
B & 0 & 21348.22 & $10(5)$ \\
C & 0 & 22080.08 & $7(4)$ \\
B1 & 21348.22 & 32683.69 & $140(50)$ \\
C2 & 22080.08 & 33352.15 & $200(40)$ \\
\hline \hline
\end{tabular}

ionic ground state may not be assigned unambiguously. Lowlying excited states in Pm II from the NIST databse [11] were also considered as Rydberg-series limits, most importantly the ${ }^{7} \mathrm{H}_{3}^{o}$ state at $446.45 \mathrm{~cm}^{-1}$, representing the state in $\mathrm{Pm}$ II with the lowest excitation energy. In this case too, no clear assignment could be made, rendering the method of Rydberg convergences inapplicable. On the other hand, many strong autoionizing transitions can be observed, which may serve as final excitation steps for efficient photoionization of Pm, e.g., for high-resolution spectroscopy on the nuclear structure in the Pm isotopic chain.

All recorded transitions and energy levels are listed in the Supplemental Material [17]. We included a data table for each excitation scheme, where all lines are listed by wavelength in descending order. The uncertainties are stated in the table captions. The above-mentioned systematic deviation in the measured ground-state transitions was eliminated in the spectra of the second excitation steps by performing scans in both directions. For the ionizing transitions, comparatively slow scanning speeds were chosen, so the systematic effect is estimated to be well below the statistical uncertainty.

Associated energy levels for the transitions are given whenever possible. We also inferred line intensities from the ion count rate on resonance. However, these should be considered as rough guidelines, as intensities in RIS are prone to interference from all involved excitation lasers. In the schemes $\mathrm{SES}_{\mathrm{A}}$, $\mathrm{SES}_{\mathrm{B}}$, and $\mathrm{SES}_{\mathrm{C}}$, where we use an arbitrary-wavelength third step for ionization, this is particularly striking because the third step may be resonant to an autoionizing transition. In order to counteract this to some extent we repeated each scan in these schemes with a different ionization laser wavelength for a more reliable estimation of the transition intensity. In the data tables we state the mean value of both intensities. The second part of the Supplemental Material comprises excited

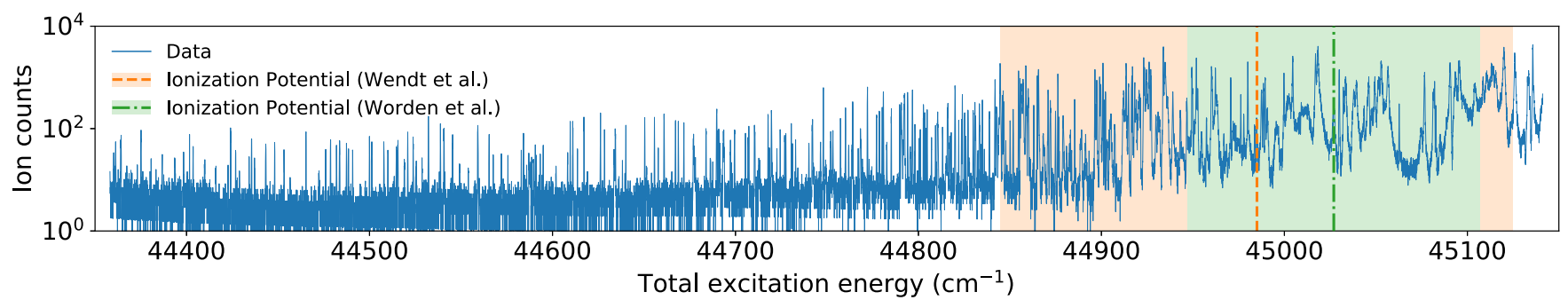

FIG. 5. Spectrum obtained from scanning the third excitation step in scheme $\mathrm{C} 2$. The literature values of the first ionization potential of $44985(140) \mathrm{cm}^{-1}$ [14] and $45027(80) \mathrm{cm}^{-1}$ [13] are shown by the orange dashed and green dash-dotted vertical lines, respectively. The shaded regions indicate the uncertainty. The ion counts are linearly scaled with respect to the laser power in the final excitation step (which varies between 0.5 and $1.3 \mathrm{~W}$ over the scan range). 
levels, sorted by energy in ascending order. The level energies were derived from the recorded transitions according to the rules stated in [24]. As discussed above, possible values for the total angular momentum $J$ are included in the data tables.

\section{IONIZATION POTENTIAL}

Our approach for the IP measurement is based on a dc electric field ionization of highly excited states. The underlying concepts, namely, tunneling of the electron through the potential barrier along the electric field axis and the classical view of the so-called saddle-point model, are discussed and compared in detail by Littman et al. [33]. With increasing field strengths tunneling leads to an exponential increase of ionization rates of excited atoms and is most notably observed through a gradual broadening of Stark sublevels into a continuum. Saddle-point ionization, on the other hand, manifests as a sharp increase of ionization rates at excitation energies greater than the saddle-point of the effective Coulomb potential, which can be written as

$$
W_{s}=\mathrm{IP}-2 \sqrt{\frac{Z_{\mathrm{eff}} e^{3} F}{4 \pi \epsilon_{0}}},
$$

with the effective charge of the atomic core $Z_{\mathrm{eff}}$ and the external electric field $F$. A precise measurement of several electric field ionization thresholds allows an extrapolation to zerofield strength, thus yielding the ionization potential. From the experimental point of view, tunneling can be neglected in this case, as ionization rates are very small at moderate electric fields and do not significantly influence the sharp saddle-point threshold. After inserting values for the constants, this law simplifies to $W_{s}=\mathrm{IP}-6.12(\mathrm{~V} \mathrm{~cm})^{-1 / 2} \sqrt{F}$ (assuming $Z_{\mathrm{eff}}=$ 1 for high excited states). At this point one should note that Eq. (1) requires a modification in the form of additional terms proportional to $\left|m_{F}\right| F^{3 / 4}+\frac{3}{16} m_{F}^{2} F$ (note that the subscript $F$ does not denote the electric field, but the total angular momentum) for states with nonzero magnetic quantum numbers $[33,34]$. A simple explanation is that energy which is stored in angular momentum perpendicular to the axis of the electric field cannot contribute to the escape of the electron over the potential barrier. Consequently, states of high $\left|m_{F}\right|$ require significantly stronger electric fields for ionization. Gallagher et al. observed this effect for Rydberg states in sodium [35]. In our experiment all laser beams are polarized perpendicular the direction of the electric field, while the first excitation step laser is also polarized perpendicular with respect to the second and third excitation step lasers. We do not selectively excite certain $m_{F}$ sublevels and thresholds are dominated by the $m_{F}=0$ contribution.

For this experiment we operate the laser ion source slightly differently than described in Sec. II. Instead of guiding all lasers along to the ion beam axis, we cross the lasers between the two flat electrodes $U_{1}$ and $U_{2}$, as depicted in Fig. 2 by a transversal laser beam. In this geometry laser ionization no longer takes place inside the atomizer. Neutral Pm atoms effuse from the furnace and the laser-atom interaction region is spatially well defined within a static electric field generated by $U_{1}$ and $U_{2}$. The electrodes are set $1 \mathrm{~cm}$ apart and have central holes of $2 \mathrm{~mm}$ diameter. In order to suppress surface ionized

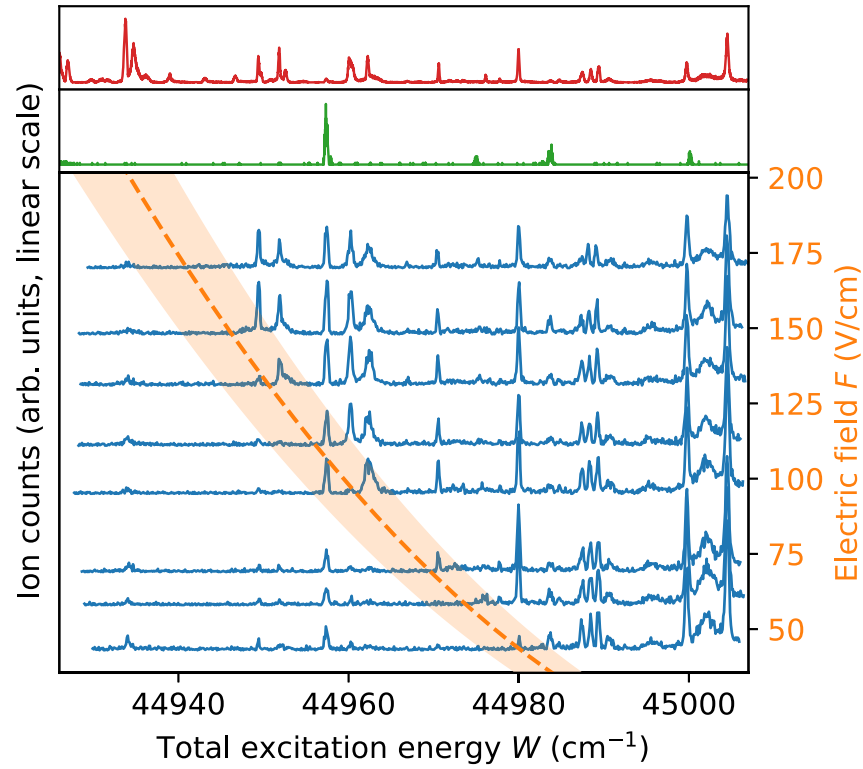

FIG. 6. Determination of ionization thresholds from scans of the final laser excitation step in scheme $\mathrm{C} 2$. The upper trace shows the full resonant in-source laser scan (red), the middle trace the in-source laser scan with detuned second excitation step, revealing parasitic resonanes (green); and the lower trace the laser ionization scans with external electric field (blue). The ion count offset for each scan is scaled with the corresponding electric field strength. The orange dashed line illustrates the electric field ionization threshold, with the shaded region indicating the uncertainty.

species from the furnace, $U_{1}$ is set on a positive voltage. Because saddle-point ionization is very similar to the mechanism of autoionization, states above the threshold ionize almost instantaneously and independently of the absolute field strengths (in contrast to tunneling). As a consequence, ions are generated with a relatively small spatial spread around the laser-atom interaction region, which is advantageous in terms of the ion beam energy spread. Nevertheless, as we tune the electric field, the voltages on the extraction electrode $U_{3}$ and the quadrupole deflector have to be adjusted in order to guarantee optimal transmission through the apparatus.

Initially we recorded the highly excited spectrum by scanning the wave number of the laser used in the final excitation step in the presence of a constant electric field, similar to the approach of Köhler et al. [26] and Erdmann et al. [27] in the actinide series.

Figure 6 shows the results for laser scans with excitation scheme $\mathrm{C} 2$ over a range of approximately $6 \mathrm{~nm}$ in the region slightly below the expected ionization potential. The upper trace shows the relevant region of the spectrum obtained from ionization inside the atomizer furnace (see Fig. 5), i.e., containing all resonances independent of the electric field. The spectra from transversal laser-atom interaction inside the electric field are presented in the lower trace. For the sake of clarity we added an offset proportional to the electric field strength. In comparison to the full spectrum, one can clearly observe that the spectra are subsequently cut off towards lower excitation energies. Strong resonances below the threshold (e.g., at approximately $44930 \mathrm{~cm}^{-1}$ ) may 

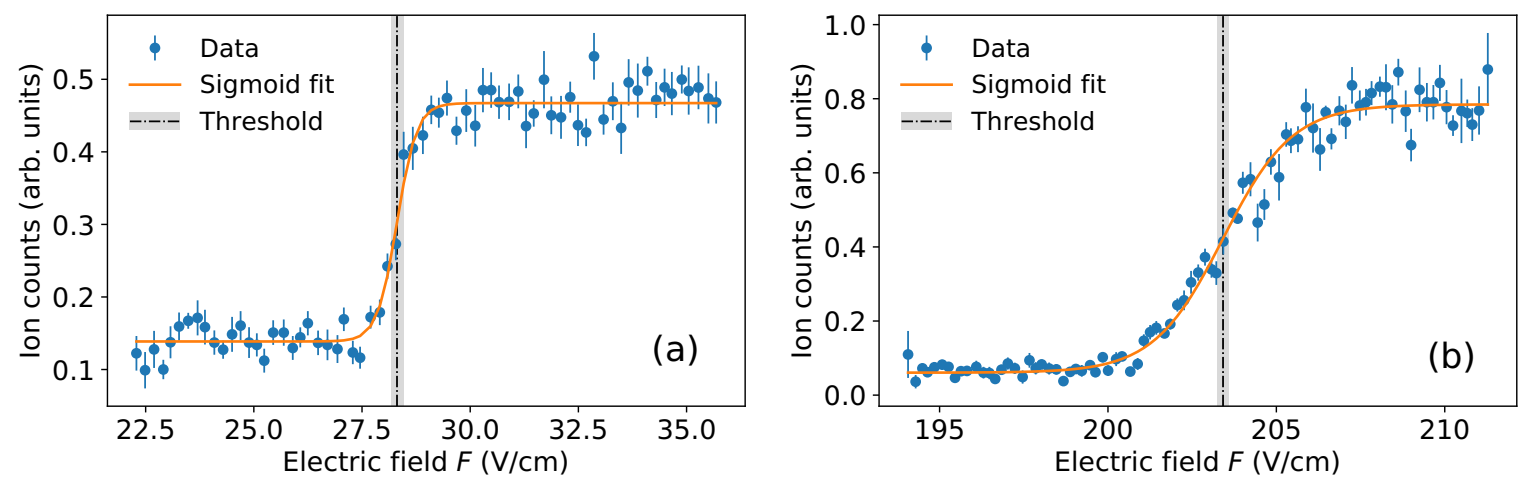

FIG. 7. Electric field ionization threshold for the energy levels at (a) $44989.3 \mathrm{~cm}^{-1}$ and (b) $44933.8 \mathrm{~cm}^{-1}$. The threshold corresponds to the turning point of the sigmoid fit. The shaded region indicates the uncertainty.

remain visible, albeit strongly suppressed, due to nonresonant photoionization or collisional ionization of the excited atoms. One resonance slightly below $44960 \mathrm{~cm}^{-1}$ is hardly affected by the electric field. Here the scanned third laser excites the $22080.08 \mathrm{~cm}^{-1} \rightarrow 33685.3 \mathrm{~cm}^{-1}$ transition, followed by nonresonant ionization $\left(v_{1}+v_{3}+v_{3}\right)$. These parasitic resonances can be identified by means of a scan with an offresonant second step, which in this case corresponds to excitation scheme $\mathrm{SES}_{\mathrm{C}}$. The relevant region of this scan is plotted in the middle trace of Fig. 6. At this energy scale, all peaks in this spectrum correspond to artifacts in scheme C2 and may be ignored with regard to ionization thresholds.

Because no clear nonresonant ionization onset can be observed, thresholds can only be constrained by the presence or absence of resonances for given electric field strengths. By comparing two data sets, we estimate the photoionization threshold as the mean value of the energy of a peak which disappeared and the next higher energy peak, with an error range spanning to either side. Depending on the local spectral level density, this leads to rather unprecise results, but nonetheless allows a first direct IP determination. With Eq. (1) we derive ${ }^{2}$

$$
W_{s}^{\lambda}=45020.2(6) \mathrm{cm}^{-1}-6.07(6)(\mathrm{V} \mathrm{cm})^{-1 / 2} \sqrt{F} \text {. }
$$

The corresponding curve and error range are included in Fig. 6. The extracted IP law meets the expectation of $W_{s} \propto$ $6.12(\mathrm{~V} \mathrm{~cm})^{-1 / 2} \sqrt{F}$; however, the achieved precision in the IP value is not satisfactory due to the strong dependence on the spectral level density. This can be improved by turning around the measurement procedure: Instead of scanning the final laser excitation step, we keep the laser on resonance and vary the electric field strength. Naturally this method does not rely on a nonresonant ionization onset and therefore counting statistics benefit from the resonant ionization process. Energy levels can be precisely assigned to electric field thresholds, determined from a sharp increase in the ion count rate. However, the improved precision comes at the cost of increased complexity in the measurement process, since knowledge of the atomic spectrum in the relevant range is a prerequisite for the applicability of this method. Moreover, the scanning procedure is

\footnotetext{
${ }^{2}$ With $W_{s}^{\lambda}$ we refer to thresholds obtained from scanning the photon energy at a fixed electric field strength, whereas $W_{s}^{F}$ are fixed energies used for scans of the electric field strength.
}

not trivial as the voltages $U_{1}$ and $U_{2}$ significantly influence the ion beam transmission through the ion optics downstream. For this reason we performed reference scans on the energy level at $45005 \mathrm{~cm}^{-1}$ (which is above threshold for all investigated values of $F$ ) for relevant voltage sets. Ionization thresholds were measured for 11 energy levels in the range from 44930 to $44990 \mathrm{~cm}^{-1}$ with electric fields between 25 and $205 \mathrm{~V} / \mathrm{cm}$. All data sets were corrected for the ion beam transmission loss with the corresponding reference scans. The resulting saddle-point ionization thresholds for the highest and lowest investigated level energies are presented in Figs. 7(a) and 7(b), respectively. The data can be well described with a sigmoid function

$$
S(F)=A_{0}+\frac{A_{1}}{1+e^{-k\left(F-F_{T}\right)}},
$$

with an offset $A_{0}$, amplitude $A_{1}$ and turning point $F_{T}$. The turning point can be identified as the electric field threshold for the corresponding resonance. For $F<F_{T}$ the high-energy tail of the resonance is gradually ionized and for $F>F_{T}$ the low-energy tail, respectively. Consequently, for symmetric line shapes $F_{T}$ corresponds to an ionization rate of $A_{0}+A_{1} / 2$. The obtained values $F_{T}$ with the associated energies $W_{s}$ are displayed in Fig. 8. A fit with Eq. (1) yields the IP law

$$
W_{s}^{F}=45020.8(3) \mathrm{cm}^{-1}-6.08(1)(\mathrm{V} \mathrm{cm})^{-1 / 2} \sqrt{F} .
$$

The extracted value of $\operatorname{IP}_{(\mathrm{Pm})}=45020.8(3) \mathrm{cm}^{-1}$ is more than one order of magnitude more precise than the one derived from $W_{s}^{\lambda}$ thresholds. The line slopes in the $W_{s}^{\lambda}$ and $W_{s}^{F}$ IP laws are in perfect agreement with each other; however, at higher precision we observe a significant deviation from the expected value of $6.12(\mathrm{~V} \mathrm{~cm})^{-1 / 2}$. Moreover, one could argue about a minor systematic trend in the fit residuals, which are presented in the insets of Fig. 8. While this does not necessarily require a modification to our fitting function (as the data are still within a $1 \sigma$ range), it motivates a careful consideration of possible systematic effects in our measurements. Merkt et al. report similar deviations (i.e., lower than expected slopes) for field ionization of Rydberg states in argon [36], which become more significant towards lower electric field strengths. They attribute this observation to electric field inhomogeneities. For our setup we simulated electric fields in the ion source region for each set of applied voltages, using the IBSIMU $\mathrm{C}++$ library [37]. The simulations yield a constant 


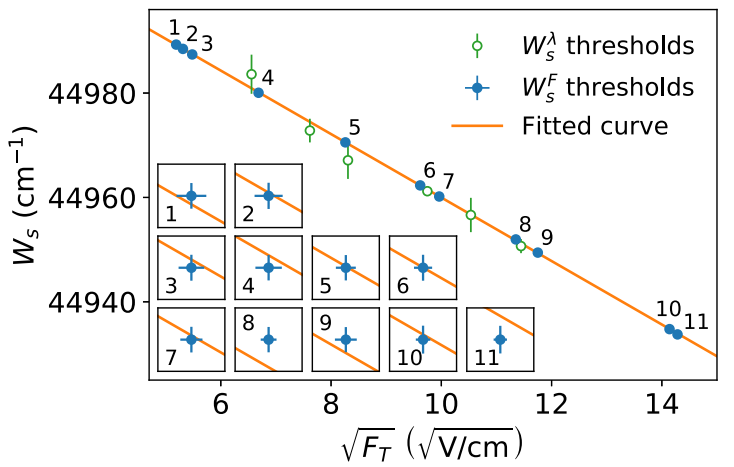

FIG. 8. Overview of all extracted ionization thresholds. The open green circles are the photoionization thresholds obtained from wavelength scans at a fixed electric field (see Fig. 6), whereas the closed blue circles are obtained from the electric field scans at a given level energy (see Fig. 7). The linear fit for the extraction of the ionization potential (solid orange line) corresponds to the latter. The insets display the deviation of the fitted curve to the data points (labeled 1-11) with a magnification factor of 27.

shift of approximately $-0.3 \%$ from the nominal field, i.e., $\left(U_{1}-U_{2}\right) \mathrm{cm}^{-1}$. This shift is already considered in the data presented above. A displacement of the laser-atom interaction region, e.g., through misalignment of the transversal laser, would induce an additional field offset due to minor electric field inhomogeneities. A shift towards the lower field region would explain the observed deviation. Further effects can be recognized in the threshold widths. We included the spectral widths $\Delta W$ in Table II, which were calculated from widths with respect to the electric field, using Eq. (3). The $\Delta W$ widths are relatively constant except for the two highest field thresholds. Three effects play a role in our observation. (i) The finite size of the laser-atom interaction region within the field gradient forces a lower limit on the width of field-induced thresholds. (ii) A slightly off-resonant excitation laser will cause a broadening and shift the threshold energy towards the excitation energy. (iii) The Stark effect causes a level splitting which broadens the thresholds at high fields. Possibly this effect can be observed in the two highest field thresholds. We expect the influence of the Stark effect to be somewhat limited to the laser linewidth, since in our measurement we use fixed

TABLE II. Electric field ionization thresholds $F_{T}$ for given excitation energies $W_{s}$, with corresponding spectral threshold widths $\Delta W$.

\begin{tabular}{lrc}
\hline \hline$W_{s}\left(\mathrm{~cm}^{-1}\right)$ & $F_{T}(\mathrm{~V} / \mathrm{cm})$ & $\Delta W\left(\mathrm{~cm}^{-1}\right)$ \\
\hline $44989.29(9)$ & $26.94(10)$ & $0.6(2)$ \\
$44988.46(9)$ & $28.23(10)$ & $0.5(2)$ \\
$44987.40(9)$ & $30.05(9)$ & $0.4(1)$ \\
$44980.06(9)$ & $44.68(12)$ & $0.5(2)$ \\
$44970.55(9)$ & $68.22(11)$ & $0.4(1)$ \\
$44962.31(9)$ & $92.48(12)$ & $0.4(1)$ \\
$44960.18(9)$ & $99.24(15)$ & $0.6(2)$ \\
$44951.96(9)$ & $128.85(12)$ & $0.5(2)$ \\
$44949.43(9)$ & $138.06(17)$ & $0.6(2)$ \\
$44934.77(10)$ & $199.92(14)$ & $0.8(2)$ \\
$44933.75(10)$ & $204.03(13)$ & $1.0(3)$ \\
\hline \hline
\end{tabular}

wavelength lasers and have to consider a convolution of the approximately Gaussian spectral laser profile and the Stark manifold as excited states. From our data we do not find a maximum width limit $\Delta W$ of the thresholds, which could be associated with the laser linewidth (which is on the order of $10 \mathrm{GHz}$ ). We expect saturation effects from the high-power pulsed excitation to extend this beyond the laser linewidth.

In addition to threshold broadening, the Stark effect may also induce systematic shifts. In the case of a hydrogenic system, blueshifted levels ionize at field strengths far beyond the saddle point, as they are located on the high-energy side of the potential [38]. Although this restriction is lifted in complex atomic systems and blueshifted states may autoionize over the underlying redshifted continuum, they require higher fields than the classical saddle-point limit for ionization (for details see [36] and references therein). This results in an offset of field-induced thresholds towards higher fields strengths. Note that states which show no splitting may also be affected by a Stark shift, which is magnified by avoided crossings of strongly interacting neighboring states [38]. A conclusive analysis of these effects is hampered by the experimental resolution in our setup and the lack of knowledge about the configuration of the investigated states. However, as one aims to push towards higher precision, all the aspects mentioned above should be taken into account.

\section{CONCLUSION}

We have presented an extensive study in the spectrum of neutral promethium. The data cover 126 odd energy levels, 546 even energy levels, and more than 1000 transitions, which add to the knowledge about $\mathrm{Pm}$ atomic structure and provide a considerable contribution to its atomic spectra database. The data may give valuable input for the study of such phenomena as quantum chaos in the exceptionally dense atomic spectrum, as recently investigated in $\mathrm{Pa}$ [25].

We developed photoionization schemes for Pm, which pave the way for efficient production of high-purity $\mathrm{Pm}$ ion beams. Possible applications include, e.g., fundamental nuclear physics research on the $\mathrm{Pm}$ isotopic chain or isobar-free ultratrace analysis via resonance ionization mass spectrometry.

The precisely measured value of the first ionization potential serves as a valuable benchmark for $a b$ initio atomic physics and quantum chemistry calculations. Our findings confirm the predictions of Worden et al. [13] and Wendt et al. [14], which were assuming a linear trend in the ionization potentials of light lanthanide elements below the half-filling of the $4 f$ shell. Our measured value of $\mathrm{IP}_{(\mathrm{Pm})}=$ $45020.8(3) \mathrm{cm}^{-1}$ perfectly fits into this trend (which is tantamount to the good agreement with the interpolation values). The improved precision of a factor of 270 or 470 compared to the previous estimates of Worden et al. and Wendt et al., respectively, could be achieved with the measurement of electric field ionization thresholds, which proves to be a powerful method for the IP determination in complex spectra. The use of narrow linewidth laser systems or dedicated field ionization ion sources, allowing for stronger and more homogeneous fields, could push the precision even further. In this case systematic influences from the Stark effect must be analyzed and considered carefully. 


\section{ACKNOWLEDGMENTS}

D. Studer gratefully acknowledges financial support from the EU through ENSAR2-RESIST (Grant No. 654002). R.H. acknowledges financial support from the Bundesministerium für Bildung und Bundesministerium fr Bil- dung und Forschung (BMBF Germany) under Grant No. 05P15UMCIA. The open access fee was covered by FILL2030, an European Union project within the European Commission's Horizon 2020 Research and Innovation Programme under Grant Agreement No. 731096.
[1] H. Flicker, J. J. Loferski, and T. S. Elleman, IEEE Trans. Electron 11, 2 (1964).

[2] M. Kumar, J. Udhayakumar, J. Nuwad, R. Shukla, C. G. S. Pillai, A. Dash, and M. Venkatesh, Appl. Radiat. Isotopes 69, 580 (2011).

[3] G. J. Beyer and T. J. Ruth, Nucl. Instrum. Methods Phys. Res., Sect. B 204, 694 (2003).

[4] H. Mohsin, F. Jia, J. N. Bryan, G. Sivaguru, C. S. Cutler, A. R. Ketring, W. H. Miller, J. Simón, R. K. Frank, L. J. Theodore, D. B. Axworthy, S. S. Jurisson, and M. R. Lewis, Bioconjugate Chem. 22, 2444 (2011).

[5] F. Rösch, Radiochimica Acta 95, 303 (2009).

[6] H. Uusijärvi, P. Bernhardt, F. Rösch, H. R. Maecke, and E. Forsell-Aronsson, J. Nucl. Med. 47, 807 (2006).

[7] W. F. Meggers, B. F. Scribner, and W. R. Bozman, J. Res. Natl. Bur. Stand. 46, 85 (1951).

[8] P. Klinkenberg and F. S. Tomkins, Physica 26, 103 (1960).

[9] J. Reader and S. P. Davis, J. Opt. Soc. Am. 53, 431 (1963).

[10] J. Reader and S. P. Davis, J. Res. Natl. Bur. Stand. A 71A, 587 (1967).

[11] A. Kramida and Y. Ralchenko, NIST Atomic Spectra Database, NIST Standard Reference Database 78 (National Institute of Standards and Technology, Gaithersburg, 2018), Version 5.6.

[12] J. E. Sansonetti and W. C. Martin, J. Phys. Chem. Ref. Data 34, 1559 (2005).

[13] E. F. Worden, R. W. Solarz, J. A. Paisner, and J. G. Conway, J. Opt. Soc. Am. 68, 52 (1978).

[14] K. Wendt, T. Gottwald, C. Mattolat, and S. Raeder, Hyperfine Interact. 227, 55 (2014).

[15] T. K. Sato, M. Asai, A. Borschevsky, T. Stora, N. Sato, Y. Kaneya, K. Tsukada, C. E. Düllmann, K. Eberhardt, E. Eliav et al., Nature (London) 520, 209 (2015).

[16] T. K. Sato, M. Asai, A. Borschevsky, R. Beerwerth, Y. Kaneya, H. Makii, A. Mitsukai, Y. Nagame, A. Osa, A. Toyoshima et al., J. Am. Chem. Soc. 140, 14609 (2018).

[17] See Supplemental Material at http://link.aps.org/supplemental/ 10.1103/PhysRevA.99.062513 for a complete list of measured transition wavelengths, level energies and uncertainties, as well as values and corresponding references for the Periodic Table in Fig. 1.

[18] C. Mattolat, T. Gottwald, S. Raeder, S. Rothe, F. Schwellnus, K. Wendt, P. Thörle-Pospiech, and N. Trautmann, Phys. Rev. A 81, 052513 (2010).

[19] J. Roßnagel, S. Raeder, A. Hakimi, R. Ferrer, N. Trautmann, and K. Wendt, Phys. Rev. A 85, 012525 (2012).

[20] S. Raeder, H. Heggen, A. Teigelhöfer, and J. Lassen, Spectrochim. Acta B 151, 65 (2019).
[21] D. A. Fink, K. Blaum, V. N. Fedosseev, B. A. Marsh, R. E. Rossel, and S. Rothe, Spectrochim. Acta B 151, 72 (2019).

[22] S. Rothe, A. N. Andreyev, S. Antalic, A. Borschevsky, L. Capponi, T. E. Cocolios, H. de Witte, E. Eliav, D. V. Fedorov, V. N. Fedosseev et al., Nat. Commun. 4, 1835 (2013).

[23] P. Chhetri, D. Ackermann, H. Backe, M. Block, B. Cheal, C. Droese, C. E. Düllmann, J. Even, R. Ferrer, F. Giacoppo et al., Phys. Rev. Lett. 120, 263003 (2018).

[24] P. Naubereit, T. Gottwald, D. Studer, and K. Wendt, Phys. Rev. A 98, 022505 (2018).

[25] P. Naubereit, D. Studer, A. V. Viatkina, A. Buchleitner, B. Dietz, V. V. Flambaum, and K. Wendt, Phys. Rev. A 98, 022506 (2018).

[26] S. Köhler, R. Deissenberger, K. Eberhardt, N. Erdmann, G. Herrmann, G. Huber, J. V. Kratz, M. Nunnemann, G. Passler, P. M. Rao, J. Riegel, N. Trautmann, and K. Wendt, Spectrochim. Acta B 52, 717 (1997).

[27] N. Erdmann, M. Nunnemann, K. Eberhardt, G. Herrmann, G. Huber, S. Köhler, J. V. Kratz, G. Passler, J. R. Peterson, N. Trautmann, and A. Waldek, J. Alloys Compd. 271-273, 837 (1998).

[28] S. Heinitz, E. A. Maugeri, D. Schumann, R. Dressler, N. Kivel, C. Guerrero, U. Köster, M. Tessler, M. Paul, and S. Halfon, Radiochim. Acta 105, 155 (2017).

[29] S. Rothe, B. A. Marsh, C. Mattolat, V. N. Fedosseev, and K. Wendt, J. Phys.: Conf. Ser. 312, 052020 (2011).

[30] V. Sonnenschein, I. D. Moore, H. Khan, I. Pohjalainen, and M. Reponen, Hyperfine Interact. 227, 113 (2014).

[31] A. Teigelhöfer, P. Bricault, O. Chachkova, M. Gillner, J. Lassen, J. P. Lavoie, R. Li, J. Meißner, W. Neu, and K. D. A. Wendt, Hyperfine Interact. 196, 161 (2010).

[32] F. Schneider, K. Chrysalidis, H. Dorrer, C. Düllmann, K. Eberhardt, R. Haas, T. Kieck, C. Mokry, P. Naubereit, S. Schmidt, and K. Wendt, Nucl. Instrum. Methods Phys. Res. Sect. B 376, 388 (2016).

[33] M. G. Littman, M. M. Kash, and D. Kleppner, Phys. Rev. Lett. 41, 103 (1978).

[34] W. E. Cooke and T. F. Gallagher, Phys. Rev. A 17, 1226 (1978).

[35] T. F. Gallagher, L. M. Humphrey, W. E. Cooke, R. M. Hill, and S. A. Edelstein, Phys. Rev. A 16, 1098 (1977).

[36] F. Merkt, A. Osterwalder, R. Seiler, R. Signorell, H. Palm, H. Schmutz, and R. Gunzinger, J. Phys. B 31, 1705 (1998).

[37] T. Kalvas, O. Tarvainen, T. Ropponen, O. Steczkiewicz, J. Arje, and H. Clark, Rev. Sci. Instrum. 81, 02 B703 (2010).

[38] T. F. Gallagher, Rep. Prog. Phys. 51, 143 (1988). 